\title{
EUFOREA Rhinology Research Forum 2017: report of the brainstorming sessions on endotype-driven treatment, patient empowerment and digital future in airways care*
}

Valerie J. Lund' ${ }^{1}$, Claire Hopkins², Cezmi Akdis³, Claus Bachert ${ }^{4}$, Jean Bous- $\quad$ Rhinology Online, Vol 1:11-19, 2018 quet ${ }^{5}$, Wytske J. Fokkens ${ }^{6}$, Sven Seys ${ }^{7}$, Laura Van Gerven ${ }^{8}$, Mubeccel Akdis', http://doi.org/10.4193/RHINoL/18.012 Ga Y. Ban ${ }^{10}$, Kristi Biswas ${ }^{11}$, Robert Böscke ${ }^{12}$, Victoria Boeva ${ }^{13}$, Giorgio W. Canonica ${ }^{14}$, José A. Castillo ${ }^{15}$, Seung K. Chung ${ }^{16}$, Jos A.M. Claes ${ }^{17}$, Leen Cools ${ }^{18}$, Gi- *Received for publication: useppe De Carlo ${ }^{19}$, Eugenio De Corso ${ }^{20}$, Michel Djandji21 ${ }^{21}$ Maria Doulaptsi22, February 12, 2018 Jef Feijenn' ${ }^{23}$, Stefania Gallo ${ }^{24}$, Simon Gane ${ }^{25}$, Philippe Gevaert ${ }^{26}$, Korneliusz Accepted: February 24, 2018 Golebski27, Stijn Halewyck ${ }^{28}$, Thomas Hummel29, Iñaki Izquierdo ${ }^{30}$, Alexandre Jagerschmidt ${ }^{31}$, Guy F. Joos ${ }^{32}$, Anette D. Kjeldsen ${ }^{33}$, Isabel Kloeck ${ }^{34}$, Michael Koennecke ${ }^{35}$, Oksana Kokorina ${ }^{36}$, Ilan Koren ${ }^{37}$, Inge Kortekaas-Krohnn ${ }^{38}$, Olga Krysko ${ }^{39}$, Basile N. Landis ${ }^{40}$, Bibi Lange ${ }^{41}$, Naomi Launders ${ }^{42}$, Jivianne Lee ${ }^{43}$, Garyfalia Lekakis ${ }^{44}$, Leda Mannent ${ }^{45}$, Katleen Martens ${ }^{38}$, Daniela Morghenti ${ }^{7}$, Joaquim Mullo ${ }^{46}$, Ruth Murray ${ }^{47}$, Dee O'Sullivann ${ }^{48}$, Carl Philpott ${ }^{49}$, Todor A. Popov $^{50}$, Emmanuel Prokopakis22, Philippe Rombaux ${ }^{51}$, Carmen Rondon ${ }^{52}$, Paul J. Rowe ${ }^{21}$, Nasim S. Seyed-Tabib ${ }^{53}$, Kristien Sleurs ${ }^{23}$, Kato J. S. Speleman ${ }^{54}$, Jurate Staikuniene ${ }^{55}$, Brecht Steelant ${ }^{38}$, Karel Talavera-Pérez ${ }^{23}$, Christian Taube ${ }^{56}$, Sanna Toppila-Salmi ${ }^{57}$, Thuy Tran-Le ${ }^{58}$, Justinas Vaitkus ${ }^{59}$, Saulius Vaitkus ${ }^{59}$, Klara Van Gool ${ }^{60}$, Anna Van Hoolst ${ }^{61}$, Ruth Verbrugge ${ }^{62}$, Benedicte Verhaeghe ${ }^{63}$, Stephan Vlaminck ${ }^{64}$, Martin Wagenmann ${ }^{65}$, Torsten Zuberbier ${ }^{66}$, Abel-Jan Tasman ${ }^{67}$, Benoit Pugin7, Peter W. Hellings ${ }^{8}$

${ }^{1}$ Professor Emeritus in Rhinology, University College London, London, United Kingdom

${ }^{2}$ Guy's and St Thomas' Hospitals, London, United Kingdom

${ }^{3}$ Director SIAF, University of Zurich, Director CK-CARE, Davos, Switzerland

${ }^{4}$ Head Upper Airways Research Laboratory (URL), University Hospital Ghent, Ghent, Belgium

${ }^{5}$ University Hospital, Montpellier, France

${ }^{6}$ Department of Otorhinolaryngology, Academic Medical Center, Amsterdam, The Netherlands

${ }^{7}$ European Forum for Research and Education in Allergy and Airway Diseases (EUFOREA), Brussels, Belgium

${ }^{8}$ Clinical Division of Otorhinolaryngology, Head and Neck Surgery, University Hospitals Leuven, Leuven, Belgium

${ }^{9}$ Swiss Institute of Allergy and Asthma Research (SIAF), Davos, Switzerland

${ }^{10}$ Department of Allergy and Clinical immunology, Ajou University School of Medicine, Suwon, Korea

${ }^{11}$ Department of Surgery, The University of Auckland, Auckland, New Zealand

${ }^{12}$ Department of Otolaryngology, Head and Neck Surgery, University of Lübeck, Lübeck ,Germany

${ }^{13}$ Department of ENT, Saint-Petersburg Medical Academy after S.Kirov, Saint-Petersburg, Russia

${ }^{14}$ Head Personalized Medicine Asthma \& Allergy Clinic, Humanitas University \& Research Hospital, Milano, Italy

${ }^{15}$ Hospital Universitario Quirón Dexeus, Barcelona, Spain

${ }^{16}$ Department of ORL-HNS, Samsung Medical Center, Sungkyunkwan University School of Medicine, Seoul, South Korea

${ }^{17}$ ENT Department, UZ Antwerpen, Edegem, Belgium

${ }^{18}$ Department of Otorhinolaryngology-Head and Neck Surgery, UZ Leuven, Belgium

${ }^{19}$ The European Federation of Allergy and Airways Diseases patients associations, Brussels, Belgium

${ }^{20}$ Agostino Gemelli Hospital Foundation, Catholic University of the Sacred Heart, Head and Neck Surgery Area, Institute of Otorhinolaryngology, Rome, Italy

${ }^{21}$ Sanofi, Bridgewater, NJ, USA 
${ }^{22}$ Department of Otorhinolaryngology, University of Crete Medical School, Heraklion, Crete, Greece

${ }^{23} \mathrm{KU}$ Leuven, Leuven, Belgium

${ }^{24}$ ENT Department, ASST Sette Laghi and University of Insubria, Varese, Italy

${ }^{25}$ Royal National Throat, Nose and Ear Hospital, London, United Kingdom

${ }^{26}$ Department of Otorhinolaryngology, Ghent University Hospital, Ghent, Belgium

${ }^{27}$ Department of Otorhinolaryngology, Department of Experimental Immunology, Academic Medical Center, Amsterdam, the Netherlands

${ }^{28}$ UZ Brussel/VUB, Brussels, Belgium

${ }^{29}$ Smell and Taste Clinic, Deptartment of Otorhinolaryngology, TU Dresden, Dresden, Germany

${ }^{30}$ International Clinical Development and Medical Advice Department, Uriach, Barcelona, Spain

${ }^{31}$ Translational Medicine and Early Development, Sanofi R\&D, Chilly-Mazarin, France

${ }^{32}$ Department of Respiratory Medicine, Ghent University Hospital, Gent, Belgium

${ }^{33}$ Department of Otorhinolaryngology-Head and Neck Surgery, Odense University Hospital, AND University of Southern Denmark

${ }^{34}$ ENT surgeon AZ Monica Antwerp, Antwerp, Belgium

${ }^{35}$ University Hospital Schleswig-Holstein, Campus Lübeck, Department of Otorhinolaryngology, Lübeck, Germany

${ }^{36}$ ENT-department, Saint-Petersburg Medical Academy after S.Kirov, Saint-Petersburg, Russia

${ }^{37}$ Rabin Medical Center, Petah Tikva, Israel

${ }^{38}$ Laboratory of Clinical Immunology, Department of Microbiology and Immunology, KU Leuven, Leuven, Belgium

${ }^{39}$ Upper Airways Research Laboratory, Ghent University, Gent, Belgium

${ }^{40}$ Rhinology-Olfactology Unit, Otorhinolaryngology Department, University Hospital of Geneva, Geneva, Switzerland

${ }^{41}$ Department of Otorhinolaryngology, Odense University Hospital, Odense, Denmark

${ }^{42}$ Respiratory Effectiveness Group, Cambridge, United Kingdom

${ }^{43}$ University of California, Los Angeles School of Medicine, Los Angeles, CA, USA

${ }^{44}$ Hopital Erasme, Otorhinolaryngology Department \& University Hospitals Leuven, Leuven, Belgium

${ }^{45}$ Sanofi, Chilly-Mazarin, France

${ }^{46}$ Hospital Clínic, IDIBAPS, Universitat de Barcelona, CIBERES, Barcelona, Catalonia, Spain

${ }^{47}$ Medscript, Department of Allergy and Clinical Immunology, Dundalk, Co. Louth, Ireland

${ }^{48}$ Director, myhealthapps.net at PatientView, London, United Kingdom

${ }^{49}$ Norwich Medical School, UEA - James Paget University Hospital, Norwich, United Kingdom

${ }^{50}$ Medical University Sofia, Sofia, Bulgaria

${ }^{51}$ Service d'oto-rhino-laryngologie, Département de neuropsychiatrie et pathologies spéciales, Cliniques universitaires Saint-Luc

${ }^{52}$ Allergy Unit, Regional University Hospital, Málaga, Spain

${ }^{53} \mathrm{PhD}$ candidate, Master of biomedical sciences, Ghent University, Ghent, Belgium

${ }^{54}$ AZ Sint-Jan Brugge Oostende AV, Brugge Belgium

${ }^{55}$ Lithuanian University of Health Sciences, Medical Academy, Department of Immunology and Allergology, Kaunas, Lithuania

${ }^{56}$ Department of Pulmonary Medicine, University Hospital Essen - Ruhrlandklinik, Essen , Germany

${ }^{57}$ Skin and Allergy Hospital, Helsinki University Hospital and University of Helsinki, Helsinki, Finland

${ }^{58}$ Thuy Tran Otolaryngology Clinic, HaNoi, Vietnam

${ }^{59}$ Department of Otorhinolaryngology, Lithuanian University of Health Sciences, Kaunas, Lithuania

${ }^{60}$ Department of Otorhinolaryngology and Head \& Neck Surgery, Antwerp University Hospital, University of Antwerp, Belgium

${ }^{61}$ UZ Leuven, Leuven, Belgium

${ }^{62}$ Allergy Laboratories Copenhagen, Copenhagen, Denmark

${ }^{63}$ Sint-Jozefskliniek, Izegem, Belgium

${ }^{64}$ AZ St-John's Hospital Bruges, Bruges, Belgium

${ }^{65}$ Department of Otorhinolaryngology, Head and Neck Surgery (HNO-Klinik), Universitätsklinikum Düsseldorf, Düsseldorf, Germany

${ }^{66}$ Department of Dermatology and Allergy, Charité, Universitätsmedizin Berlin, Berlin, Germany

${ }^{67}$ Rhinologie, Plastische Gesichtschirurgie, Hals-Nasen-Ohrenklinik, Kantonsspital St. Gallen, Switzerland 


\begin{abstract}
The second European Rhinology Research Forum organized by the European Forum for Research and Education in Allergy and Airway Diseases (EUFOREA) was held on 9-10th November 2017, combined with a specific symposium on air pollution and mobile Health technology (mHealth) with the GARD (Global Alliance against Chronic Respiratory Diseases) initiative of WHO (World Health Organization). Physicians from different specialties, researchers, as well as patients and industry representatives from more than 40 countries took part in the Forum. Relevant topics were debated with the aim of allowing the implementation of precision medicine (PM) in daily respiratory care. All debates started with positioning the current state of the art: identification of current gaps in practice, the current consensus and the need for implementation of novel approaches such as endotype-driven treatment, patient empowerment and eHealth tools.

This report provides a summary of the outcomes of the brainstorming sessions of the European Rhinology Research Forum 2017, highlighting the research needs in PM, with personalized care, prediction of success of treatment, participation of the patient and prevention of disease as key drivers for improving current clinical practice.
\end{abstract}

Key words: endotype, eHealth, patient empowerment, rhinitis, rhinosinusitis, precision medicine

\section{Introduction}

The second European Rhinology Research Forum (www.rhinologyresearch.eu) organized by the European Forum for Research and Education in Allergy and Airway Diseases (EUFOREA) was held under the auspices of the European Rhinologic Society, the Benelux Rhinologic Society, and the Global Allergy and Asthma European Network (GA2LEN) (1).

\section{Structure and methodology of the meeting}

The meeting was organized to address unmet needs in the field of rhinology and related allergic diseases and to explore novel possibilities for better management and patient care with emerging technologies. Allergic diseases, especially inhalant allergens, are still on the rise and pose a serious socioeconomic health problem. Estimates calculate that 50 to 100 billion Euros annually could be saved with better and earlier intervention (2). One of the problems identified in these studies is a clear undertreatment due to low adherence and the very high inter-individual variability of responsible trigger factors.
Following state-of-the art lectures on the latest research in the field of rhinitis, rhinosinusitis, asthma and chronic obstructive pulmonary disease (COPD), clinicians, researchers and patient representatives participated in brainstorming sessions on the future landscape in respiratory care (Figure 1). The objective of these sessions was to discuss the implementation of Precision Medicine (PM) into daily care, with personalized care, prediction of success of treatment, participation of the patient and prevention of disease as the major drivers for improving current clinical practices ${ }^{(3)}$. Research in the field of PM in respiratory care is supported by EUFOREA, paving the way for a more structured scientific approach for patients with chronic airway inflammation.

The 3 topics for brainstorming were proposed on the basis of the current evolutions in health care:

1) Obstacles and strategies for endotype-driven treatment for chronic rhinosinusitis (CRS). Endotype-driven treatment targeting direct pathophysiological mechanisms of the disease has become very appealing for both patients and doctors. This novel
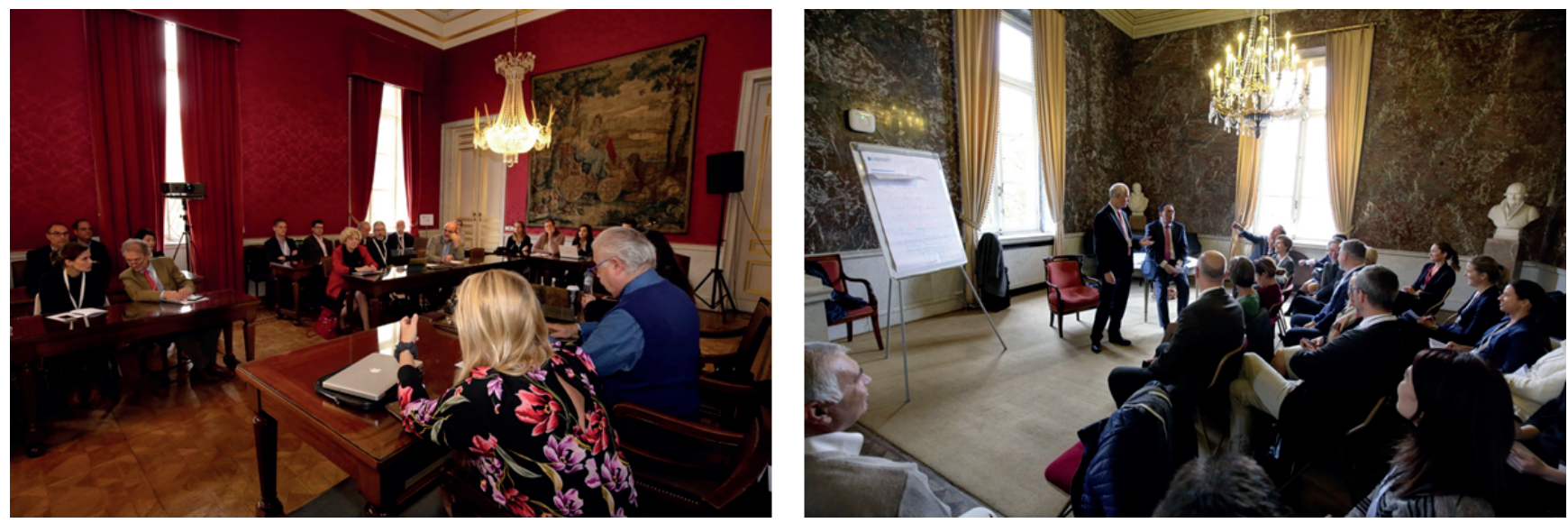

Figure 1. Impressions of the brainstorming sessions 


\section{Who?}

- Uncontrolled CRS patients

- 'Severe' phenotypes : CRS patients with multimorbidities (e.g. asthma or N-ERD)

\section{Where?}

- Reference centres for biologicals all over Europe

- Focus on overlapping and mixed endotypes

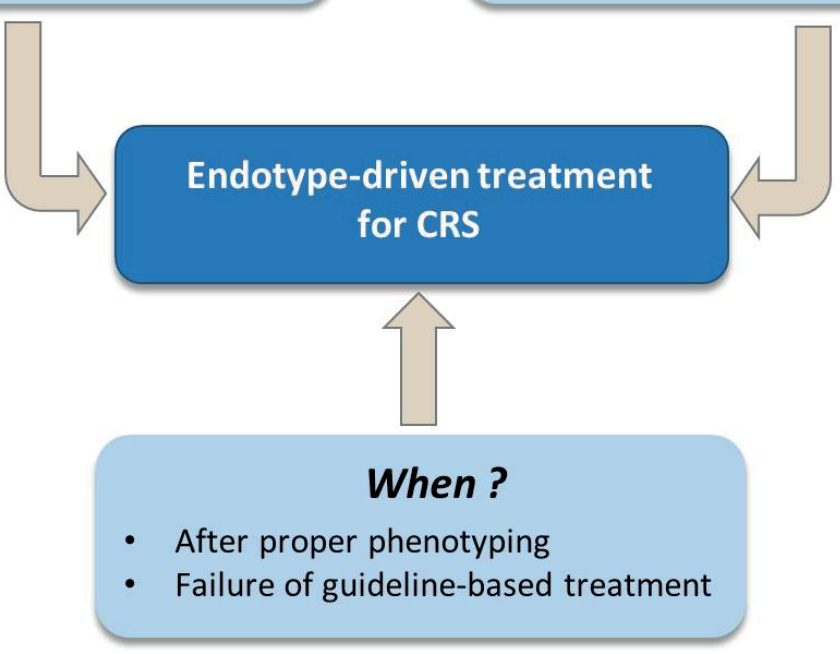

Figure 2. Obstacles and strategies for endotype-driven treatment for CRS.

approach is attractive given its targeted approach with better patient-reported outcomes of treatment ${ }^{(4)}$ and should encompass a proper diagnosis ${ }^{(5,6)}$, including multimorbidities ${ }^{(7,8)}$ and eventually local allergic reaction ${ }^{(9)}$.

2) Guidelines empowering patients. Patient empowerment is a health care priority for the EU Commission, and supported by EUFOREA ${ }^{(10)}$.

3) Digital future for airways care. Novel eHealth tools for patient empowerment are considered an elegant way forward in increasing patient empowerment, achieving better control of disease and secondary and tertiary prevention.

The aim of the brainstorming sessions was to discuss the current gaps in care pathways and define research priorities in the field of respiratory care.

\section{Obstacles and strategies for endotype-driven treat- ment for CRS}

A non-negligible proportion of CRS patients remain uncontrolled with current treatment options, with a high recurrence rate after medical therapy and endoscopic sinus surgery ${ }^{(11,12)}$. Guidelines for CRS treatment recommend anti-inflammatory treatment (mainly steroids), supplemented by surgery in case of failure ${ }^{(13)}$. The limited treatment options for both CRS with (CRSwNP) and without (CRSsNP) nasal polyps, coupled with the high failure rate of maximal medical therapy and even surgery, are driving the medical community to explore novel promising treatment options such as biologicals to better control the disease ${ }^{(1,3)}$. The use of biologicals is currently under investigation for CRSwNP with and without multimorbidities comprising
Type 2 inflammation, with very promising results and hope for a better control ${ }^{(14)}$. Type 2 inflammation involve specific CD4 ${ }^{+}$ T cells known as Th2 cells or non-T cell receptor carrying innate lymphoid cells type 2 that both secrete IL-4, IL-5, and IL-13 and stimulate Type 2 immunity, characterized by high IgE antibody titers and eosinophilia. However, despite the promise of endotype-driven treatment in the context of Precision Medicine, treatment with novel biologicals is associated with obstacles for implementation in real life ${ }^{(3)}$.

\section{Obstacles for endotype-driven treatment for CRSwNP}

The main obstacles for endotype-driven treatment are questions related to when, who and where to endotype (Figure 2). Not all CRS patients need to be "endotyped" at their first visit because of practical considerations and optimal disease control achieved with first-line treatment in a large group of patients. Endotyping is used more frequently by those physicians working in tertiary referral centres where the majority of patients have already received medical therapy and/or surgery that had failed to control the disease.

Discussion during the forum acknowledged the need to trade off what is desirable, affordable and sustainable. During the first visit, the focus should be on good phenotyping of the patient using sinonasal symptoms, nasal endoscopy and imaging CT scan if necessary, as well as severity and control of disease, rather than on endotyping ${ }^{(13)}$. If a CRS patient is well phenotyped and treated but remains uncontrolled, then a good work-up for immunologic endotyping is proposed. Besides the CRS patients with uncontrolled disease, there was 


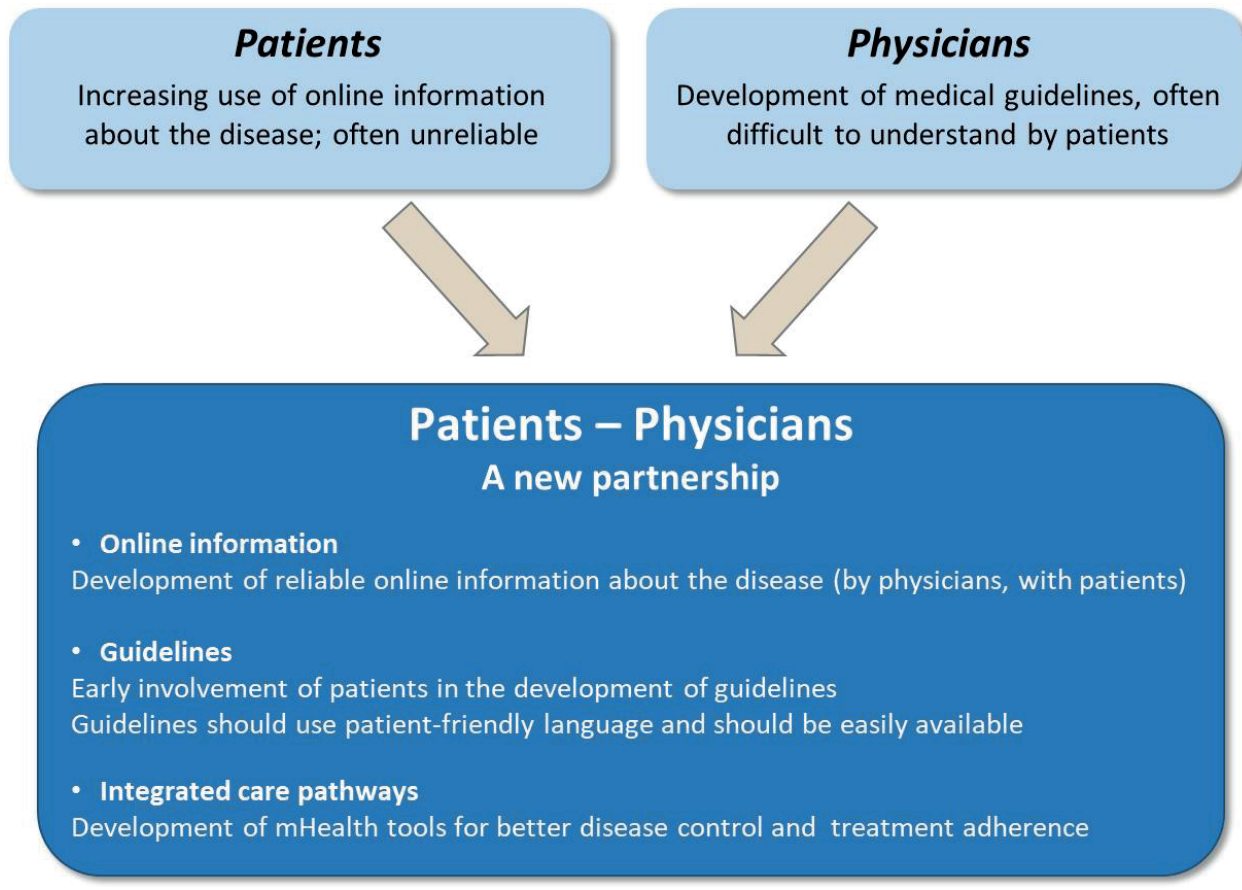

Figure 3. Guidelines empowering patients.

consensus to endotype particularly more severe phenotypes of CRS, such as patients with multimorbidities like allergy, asthma and immune deficiencies ${ }^{(15)}$. Indeed, a high likelihood of disease progression is found in CRS patients with concomitant asthma and NSAID-exacerbated respiratory disease (N-ERD) ${ }^{(16)}$. If this disease progression could be modulated or arrested by biological treatment, endotyping is of utmost importance in the severe CRS patient population from the early stages of disease. Again it was recognized that many patients with CRS will respond to current therapeutic regimens and would not likely be candidates for biological therapies. Those with aspirin intolerance, nasal polyposis and asthma (APA) triad and other severe phenotypes have the highest rates of healthcare utilization related to uncontrolled disease, and endotyping would be more cost-effective, if it were able to identify those with a poor prognosis, who are likely to undergo multiple surgeries, and facilitate the use of disease modifying agents at an early stage.

The lack of sensitive and specific biomarkers to identify different endotypes of CRSwNP is considered a major challenge to implement endotyping into clinical practice. Different sampling locations and methods (tissue, nasal secretion, serum) and different markers may be measured (lgE, eosinophil levels, periostin, IL5, IL13, IL33 and others) ${ }^{(17,18)}$. Ease of testing, cost, and availability of the sample must also be considered.

Finally, reference centres for the administration of biologicals will be required to optimize the treatment, lower the costs and share experience and data collection. This will allow greater expertise to develop in addressing overlapping or complex endotypes that are a challenge to treat. Another goal of reference centres across Europe should be to collect data and tissue to endotype and define the "non-responders" to biologicals, which is estimated to be a group of $20 \%$ of the treated patients. Pharmaceutical companies may be less motivated to identify who should not receive biological agents, but this will be valuable work to optimize clinical and cost effectiveness.

\section{Strategies}

To establish the implementation of endotyping CRS patients in daily practice, scientific evidence should be developed to convince the health care system and associated organizations of the cost-effectiveness of this method. Therefore, solid data about the pharmaco-economics of CRS (without biologicals) are needed, including the cost of the 'complications' of both medical treatment (for example side effects of oral steroids) and endoscopic sinus surgery ${ }^{(19,20)}$. Additionally, the stress and cost of anosmia/hyposmia in CRS patients and the potential influence on their mental health ${ }^{(21,22)}$ should be evaluated in this cost estimate, since biologicals seem to have superior results on the improvement of olfaction compared to conventional therapies (14).

For the initial implementation, unanimity was achieved on biologicals being reserved for CRS patients with uncontrolled disease ${ }^{(23)}$. However, some experts argue that biological treatment might alter disease progression, giving this treatment a potential role in the 'prevention' of CRS patients to evolve towards a more severe phenotype (e.g. dupilumab as disease 


\section{Digital future in airways care - Promising technologies}
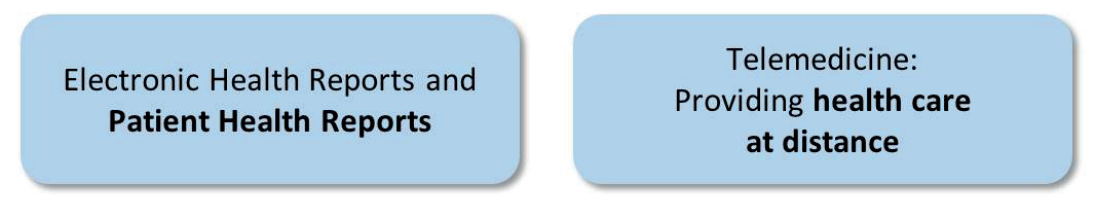

Mobile Health technology: Towards patient empowerment

\begin{tabular}{|c|c|c|c|}
\hline \multirow[b]{2}{*}{ Big data analysis } & \multicolumn{2}{|c|}{ CHALLENGES } & \multirow[b]{2}{*}{$\begin{array}{l}\text { Patient perspective: } \\
\text { - } \quad \text { Value of the App } \\
\text { - } \quad \text { Credibility of the App } \\
\text { - } \quad \text { Privacy protection }\end{array}$} \\
\hline & $\begin{array}{l}\text { GDPR compliance: } \\
\text { digital security and privacy }\end{array}$ & $\begin{array}{l}\text { Co-morbid patient: different } \\
\text { apps for different diseases }\end{array}$ & \\
\hline \multicolumn{4}{|c|}{ POTENTIALSOLUTIONS } \\
\hline $\begin{array}{l}\text { Turning big data into } \\
\text { new knowledge }\end{array}$ & Privacy Impact Assessment & $\begin{array}{l}\text { Multi-stakeholder approach } \\
\text { for co-morbid patients: } \\
\text { towards integrated apps }\end{array}$ & $\begin{array}{l}\text { Patient-centered approach: } \\
\text { - } \quad \text { Patient \& doctor feedback } \\
\text { - } \quad \text { Patient education }\end{array}$ \\
\hline
\end{tabular}

Figure 4. Digital future for airways care.

modifier) ${ }^{(14)}$. From this perspective, full endotyping of all CRS patients might become cost-effective in the future.

Finally, if endotyping and biological treatment evolve to the next level of being implemented in general clinical practice, adaptation of the EPOS guidelines with integration of the new biologicals in the treatment algorithms will be needed, taking into account regional differences reflecting the variation in organization and funding of health care systems across Europe. The non-profit organization, EUFOREA has undertaken this role to emphasize the disease burden and societal impact of CRS and multimorbidities in Europe and beyond.

\section{Guidelines empowering patients}

Patients rely on their physicians to define a therapeutic strategy and expect their physician to be aware of the guidelines. Guidelines like ARIA ${ }^{(24)}$ and EPOS ${ }^{(13)}$ are elaborated by experts and with patients' input during their development. In that context, guidelines are empowered by patients, and appreciated by the medical community and health authorities. Implementation of guidelines into clinical practice is a challenge as not all guidelines are considered as adaptable for precision medicine approaches, do not cover regional and race differences, and do not seem applicable for implementation in real life situations. In addition to providing physicians with guidance on disease management, guidelines may also empower patients to get more information on treatment options and strategies for achieving control of disease, cure and/or secondary or tertiary prevention through education. It is worth noting that patients' adherence to treatment is increased if the therapy match patient preference and beliefs ${ }^{(25)}$. Patients might receive information on the guidelines from their physicians, through the internet and/ or other channels of information.

- Physicians are considered a trustworthy source of information, despite the lack of verification of knowledge of the guidelines by most patients, and the lack of certification of physicians on guideline knowledge. For physicians, background knowledge on evidence-based treatment algorithms in guidelines is crucial for medical practice, but it appears clear that treatment algorithms need to be implemented in daily practice according to the history, needs and preferences of the patient, in alignment with the principles of precision medicine ${ }^{(3)}$.

- Online educational material on CRS and allergic rhinitis (AR) is limited and often considered unreliable, as no quality check is provided. Patient age, medical history and individual preferences might determine to what extent online information is accessed by patients. In addition, the concept and language of guidelines are common for physicians but not familiar to patients, and no patient-friendly guidelines exist.

As an outcome of discussions for empowering patients through guidelines to achieve better disease control, the debate participants support the following action plan for implementation through the EUFOREA network (Figure 3):

- Transformation of guidelines into a patient-friendly language, taking into account regional differences. Widespread 
availability of these patient guidelines should be ensured by online dissemination throughout the patient community via social media, the EUFOREA channels and patient organizations. It was considered likely that patients may become more compliant and have better outcomes when they are fully informed about the guidelines of treatment and therapeutic strategy.

- Establishment of a guideline platform accessible for both patients and physicians, allowing positioning of personalized therapeutic strategies into the treatment algorithms. The clear aims of all therapeutic strategies should be clarified: control, cure, and/or prevention.

- Support of Integrated Care Pathways, with all medical stakeholders being involved in the care pathways of CRS and rhinitis patients, through mobile (m)Health technology. From that perspective, mySinusitisCoach ${ }^{(26)}$ for CRS and Allery Diary for AR ${ }^{(27)}$ may be good tools to facilitate better patient education on guidelines, better disease control and treatment adherence, and to answer the most frequently asked questions by patients.

\section{Digital future for airways care}

\section{Current state-of-the-art}

Digital technologies are revolutionizing current health care systems ${ }^{(28)}$. Electronic health reports are used to log the patients' history, which enables easy and fast data-sharing across different hospitals and healthcare providers. More recent advances extend one step further and integrate these systems into personal health records that are accessible from the patients' smartphones ${ }^{(29)}$. These types of data-sharing allow clinicians to set up telemedicine services, a new approach to deliver health care at a distance ${ }^{(30)}$. The biggest step forward into digital health is likely to be the development of mobile health tools ${ }^{(24,31)}$. These tools allow disease monitoring via self-reported questionnaires or connection to smart sensors, tracking of medication use, patient education or mobile phone-based interventions ${ }^{(27,32,33)}$.

The promise of digital tools in chronic respiratory diseases Many mobile applications (apps) have been created and some are being implemented into daily clinical practice. For chronic respiratory diseases, most apps have been developed for lower airway diseases such as asthma or COPD ${ }^{(34)}$. The myAirCoach project was initiated to take advantage of home-monitoring and mHealth systems to predict deterioration of asthma control and occurrence of asthma exacerbations ${ }^{(35)}$. Smart sensors on inhaler devices are used to track patient adherence to asthma therapy ${ }^{(36)}$.

For allergic rhinitis, Allergy Diary by MACVIA was developed by medical experts as a mHealth tool for daily monitoring of symptoms and patient feedback about disease control (27). It follows the good practice development proposed by the EU ${ }^{(37)}$.
This information can be shared with the physician at the time of consultation and might thereby facilitate the interaction between the physician and the patient. The first validation of the app demonstrated the usefulness of the visual analogue scale (VAS) to monitor AR disease control ${ }^{(38-40)}$. mySinusitisCoach, a mobile application for patients with CRS was launched during the European Rhinology Research Forum (ERRF) $2017^{(26)}$. This app combines symptom monitoring, including the loss of smell, patient and physician feedback and patient education. Medical experts involved in the EPOS Guidelines collaborated with patients, primary care physicians and pharmacists to compile feedback during design and development of the app.

All of the above-mentioned mHealth tools are developed to improve patient empowerment and will hopefully contribute to better patient adherence, quality feedback to the physician and improved health literacy of the patient. During the brainstorming, several unmet patient needs were identified (Figure 4):

1. Guidance on the value and functionalities of the app

2. Information about app certification, data security and privacy policy

3. Increase credibility of the app by involving patient organization or advocacy group during implementation

4. Integration of apps for the co-morbid patient.

Mobile health tools provide the scientific community with a huge set of clinical data. This opens up new avenues for real-life studies but also comes with some new challenges. Firstly, the privacy of the patients should be guaranteed at all times. The EU General Data Protection Regulation (GDPR) comes into force in May 2018 to protect the privacy of EU citizens (Available from: www.eugdpr.org). Secondly, self-reported information by patients should be handled with care but provides at the same time valuable information about disease impact on the patients' quality of life. Lastly, big data sets are a challenge to analyze and a priori hypotheses are needed to avoid data mining. In contrast, big data sets have the advantage of providing new insights from combining data. Artificial Intelligence to be developed with Europe-wide or World-wide big data collections of CRS patients is expected to bring new dimensions to patient care and pharmaco-economics.

Quality of the data is not only related to the patients' input but also to the app itself. Thus far, no specific quality label for mHealth apps is in place ${ }^{(41)}$, although there are sites establishes by clinicians and other experts which evaluate apps for clinical safety and quality, such as Medappcare and dmdSanté in France, HealthOn in Germany, iMedicalApps in the US, and Our Mobile Health worldwide. The patient still has to rely mainly on the user app rating system available from the app stores, but there are curation sites, such as myhealthapps, which only feature apps tried and reviewed (and in some cases developed) by patients' associations. An attractive alternative could be the installa- 
tion of a peer-reviewing committee composed of developers of eHealth apps. It is, however, possible to certify the apps as medical devices.

\section{Conclusion}

The brainstorming sessions of the second European Rhinology Research Forum 2017 highlighted the multiple obstacles to implement effective endotype-driven treatment pointing towards the need both for better treatment of CRSwNP and multimorbidities, and the need to identify novel biomarkers with predictive value. The current AR and CRS guidelines should be tailored to the language and the needs of the patient, empowering them to become an active partner of the therapeutic strategy. Novel eHealth tools like mySinusitisCoach (CRS) and Allergy Diary (AR) pave the way to empower patients' active participation in the decision-making process of the therapeutic plan.
The third edition of the European Rhinology Research Forum will be held in November 2018 under the theme 'Immunomodulation in Respiratory Diseases' (www.rhinologyresearch.eu).

\section{Acknowledgements}

Particular thanks to Benoit Pugin for his help with the manuscript.

\section{Authorship contribution}

VL, CH, SS, LVG, BP and PWH wrote the manuscript. All other authors have contributed to the brainstorming sessions and have reviewed the manuscript.

\section{Conflict of interest}

The authors have no conflicts of interest to declare.

\section{References}

1. Hellings PW, Akdis CA, Bachert C, Bousquet J, Pugin B, Adriaensen G, et al. EUFOREA Rhinology Research Forum 2016: report of the brainstorming sessions on needs and priorities in rhinitis and rhinosinusitis. Rhinology. 2017 Sep 1;55(3):202-10.

2. Zuberbier T, Lötvall J, Simoens S, Subramanian SV, Church MK. Economic burden of inadequate management of allergic diseases in the European Union: a GA(2) LEN review. Allergy. 2014 Oct;69(10):1275-9.

3. Hellings PW, Fokkens WJ, Bachert C, Akdis CA, Bieber T, Agache I, et al. Positioning the principles of precision medicine in care pathways for allergic rhinitis and chronic rhinosinusitis - A EUFOREA-ARIA-EPOSAIRWAYS ICP statement. Allergy. 2017 Sep;72(9):1297-305.

4. De Greve G, Hellings PW, Fokkens WJ, Pugin B, Steelant B, Seys SF. Endotype-driven treatment in chronic upper airway diseases. Clin Transl Allergy. 2017;7:22.

5. Alessandri C, Ferrara R, Bernardi ML, Zennaro D, Tuppo L, Giangrieco I, et al. Diagnosing allergic sensitizations in the third millennium: why clinicians should know allergen molecule structures. Clin Transl Allergy. 2017;7:21.

6. Navarro AM, Delgado J, Muñoz-Cano RM, Dordal MT, Valero A, Quirce S, et al. Allergic respiratory disease $(A R D)$, setting forth the basics: proposals of an expert consensus report. Clin Transl Allergy. 2017;7:16.

7. Cingi C, Gevaert P, Mösges R, Rondon C, Hox V, Rudenko M, et al. Multimorbidities of allergic rhinitis in adults: European Academy of Allergy and Clinical Immunology Task Force Report. Clin Transl Allergy. 2017;7:17.

8. Anto JM, Bousquet J, Akdis M, Auffray C, Keil T, Momas I, et al. Mechanisms of the Development of Allergy (MeDALL): Introducing novel concepts in allergy phenotypes. J Allergy Clin Immunol. 2017 Feb;139(2):388-99.

9. Incorvaia C, Fuiano N, Martignago I, Gritti BL, Ridolo E. Local allergic rhinitis: evolution of concepts. Clin Transl Allergy. 2017;7:38.

10. Hellings PW, Borrelli D, Pietikainen S, Agache I, Akdis C, Bachert C, et al. European Summit on the Prevention and Self-Management of Chronic Respiratory Diseases: report of the European Union Parliament Summit (29 March 2017). Clin Transl Allergy. 2017;7:49.

11. Noon E, Hopkins C. Review article: outcomes in endoscopic sinus surgery. BMC Ear Nose Throat Disord. 2016;16:9.

12. van der Veen J, Seys SF, Timmermans $M$, Levie P, Jorissen M, Fokkens WJ, et al. Reallife study showing uncontrolled rhinosinusitis after sinus surgery in a tertiary referral centre. Allergy. 2017 Feb;72(2):282-90.

13. Fokkens WJ, Lund VJ, Mullol J, Bachert C, Alobid I, Baroody F, et al. European Position Paper on Rhinosinusitis and Nasal Polyps 2012. Rhinol Suppl. 2012 Mar;(23):3 p preceding table of contents, 1-298.

14. Bachert C, Mannent L, Naclerio RM, Mullol J, Ferguson BJ, Gevaert P, et al. Effect of Subcutaneous Dupilumab on Nasal Polyp Burden in Patients With Chronic Sinusitis and Nasal Polyposis: A Randomized Clinical Trial. JAMA. 2016 Feb 2;315(5):469-79.

15. Jarvis D, Newson R, Lotvall J, Hastan D, Tomassen $\mathrm{P}$, Keil T, et al. Asthma in adults and its association with chronic rhinosinusitis: the GA2LEN survey in Europe. Allergy. 2012 Jan;67(1):91-8.

16. Mullol J, Picado C. Rhinosinusitis and nasal polyps in aspirin-exacerbated respiratory disease. Immunol Allergy Clin North Am. 2013 May;33(2):163-76.

17. Tomassen P, Vandeplas G, Van Zele T, Cardell L-O, Arebro J, Olze H, et al. Inflammatory endotypes of chronic rhinosinusitis based on cluster analysis of biomarkers. J Allergy
Clin Immunol. 2016 May;137(5):1449-1456. e4.

18. Jonstam K, Westman M, Holtappels G, Holweg CTJ, Bachert C. Serum periostin, $\operatorname{lgE}$, and SE-lgE can be used as biomarkers to identify moderate to severe chronic rhinosinusitis with nasal polyps. J Allergy Clin Immunol. 2017 Dec;140(6):1705-1708.e3.

19. Suzuki S, Yasunaga H, Matsui H, Fushimi K, Kondo K, Yamasoba T. Complication rates after functional endoscopic sinus surgery: analysis of 50,734 Japanese patients. The Laryngoscope. 2015 Aug;125(8):1785-91.

20. Rudmik L. Economics of Chronic Rhinosinusitis. Curr Allergy Asthma Rep. 2017 Apr;17(4):20.

21. Taalman H, Wallace C, Milev R. Olfactory Functioning and Depression: A Systematic Review. Front Psychiatry. 2017:8:190.

22. Croy I, Hummel T. Olfaction as a marker for depression. J Neurol. 2017 Apr;264(4):631-8.

23. Willson TJ, Naclerio RM, Lee SE. Monoclonal Antibodies for the Treatment of Nasal Polyps. Immunol Allergy Clin North Am. 2017 May;37(2):357-67.

24. Bousquet J, Hellings PW, Agache I, Bedbrook A, Bachert C, Bergmann KC, et al. ARIA 2016: Care pathways implementing emerging technologies for predictive medicine in rhinitis and asthma across the life cycle. Clin Transl Allergy. 2016;6:47.

25. Brandstetter $S$, Finger T, Fischer W, Brandl M, Böhmer $M$, Pfeifer $M$, et al. Differences in medication adherence are associated with beliefs about medicines in asthma and COPD. Clin Transl Allergy. 2017;7:39.

26. Seys S, Bousquet J, Bachert C, Fokkens W, Agache I, Bernal-Sprekelsen M, et al. mySinusitisCoach: patient empowerment in chronic rhinosinusitis using mobile technology. Rhinology. 2018;(in press).

27. Bousquet J, Schunemann HJ, Fonseca J, Samolinski B, Bachert C, Canonica GW, et al. MACVIA-ARIA Sentinel Network for allergic 
rhinitis (MASK-rhinitis): the new generation guideline implementation. Allergy. 2015 Nov;70(11):1372-92.

28. Bousquet J, Chavannes NH, Guldemond N, Haahtela T, Hellings PW, Sheikh A. Realising the potential of mHealth to improve asthma and allergy care: how to shape the future. Eur Respir J. 2017 May;49(5).

29. Ryu B, Kim N, Heo E, Yoo S, Lee K, Hwang $\mathrm{H}$, et al. Impact of an Electronic Health Record-Integrated Personal Health Record on Patient Participation in Health Care: Development and Randomized Controlled Trial of MyHealthKeeper. J Med Internet Res. 2017 Dec 7;19(12):e401.

30. Elliott T, Shih J, Dinakar C, Portnoy J, Fineman S. American College of Allergy, Asthma \& Immunology Position Paper on the Use of Telemedicine for Allergists. Ann Allergy Asthma Immunol Off Publ Am Coll Allergy Asthma Immunol. 2017 Dec;119(6):512-7.

31. Bonini M. Electronic health (e-Health): emerging role in asthma. Curr Opin Pulm Med. 2017 Jan;23(1):21-6.

32. Ryan D, Price D, Musgrave SD, Malhotra S, Lee AJ, Ayansina D, et al. Clinical and cost effectiveness of mobile phone supported self monitoring of asthma: multicentre randomised controlled trial. BMJ. $2012 \mathrm{Mar}$ 23;344:e1756.

33. Melvin E, Cushing A, Tam A, Kitada $R$, Manice $M$. Assessing the use of
BreatheSmart ${ }^{\circledast}$ mobile technology in adult patients with asthma: a remote observational study. BMJ Open Respir Res. 2017;4(1):e000204.

34. Martínez-Pérez B, de la Torre-Díez I, LópezCoronado M. Mobile health applications for the most prevalent conditions by the World Health Organization: review and analysis. J Med Internet Res. 2013 Jun 14;15(6):e120.

35. Honkoop PJ, Simpson A, Bonini M, SnoeckStroband JB, Meah S, Fan Chung K, et al. MyAirCoach: the use of home-monitoring and mHealth systems to predict deterioration in asthma control and the occurrence of asthma exacerbations; study protocol of an observational study. BMJ Open. 2017 Jan 24:7(1):e013935.

36. Kikidis D, Konstantinos V, Tzovaras D, Usmani OS. The Digital Asthma Patient: The History and Future of Inhaler Based Health Monitoring Devices. J Aerosol Med Pulm Drug Deliv. 2016;29(3):219-32.

37. Bousquet J, Onorato GL, Bachert C, Barbolini M, Bedbrook A, Bjermer L, et al. CHRODIS criteria applied to the MASK (MACVIA-ARIA Sentinel NetworK) Good Practice in allergic rhinitis: a SUNFRAIL report. Clin Transl Allergy. 2017;7:37.

38. Caimmi D, Baiz N, Tanno LK, Demoly P, Arnavielhe S, Murray R, et al. Validation of the MASK-rhinitis visual analogue scale on smartphone screens to assess allergic rhinitis control. Clin Exp Allergy J Br Soc Allergy
Clin Immunol. 2017 Dec;47(12):1526-33.

39. Bousquet J, Bewick M, Arnavielhe S, Mathieu-Dupas E, Murray R, Bedbrook A, et al. Work productivity in rhinitis using cell phones: The MASK pilot study. Allergy. 2017 Oct;72(10):1475-84.

40. Bousquet J, Caimmi DP, Bedbrook A, Bewick M, Hellings PW, Devillier P, et al. Pilot study of mobile phone technology in allergic rhinitis in European countries: the MASKrhinitis study. Allergy. 2017 Jun;72(6):85765.

41. Sharp M, O'Sullivan D. Mobile Medical Apps and mHealth Devices: A Framework to Build Medical Apps and mHealth Devices in an Ethical Manner to Promote Safer Use - A Literature Review. Stud Health Technol Inform. 2017;235:363-7.

\section{Prof. Valerie J. Lund}

Professor Emeritus in Rhinology

University College London

London

United Kingdom

E-mail:

v.lund@ucl.ac.uk 